\title{
An Automatic Indoor Positioning Robot System Using Panorama Feature Matching
}

\author{
Jun-Wei Chang ${ }^{1}$, Huan Chen ${ }^{2}$, Krishna Kambhampaty ${ }^{3}$, Bo-Chao Cheng ${ }^{1}$ \\ ${ }^{1}$ Department of Communications Engineering, \\ National Chung Cheng University, \\ Chia-I, Taiwan 621 \\ ${ }^{2}$ Department of Computer Science and Engineering \\ National Chung Hsing University, \\ Taichung, Taiwan 402 \\ ${ }^{3}$ Department of Computer Science \\ York College of Pennsylvania \\ York, PA 17403-3651
}

\begin{abstract}
With Location-Based Service (LBS) support, a mobile user can enjoy specific information/services based on their location. The GPS-based positioning technique plays an essential role in outdoor LBS applications. Owing to physical barriers and potential interference inside a building, GPS-based positioning systems do not work well for commercial indoor LBS applications. Various wireless technology-based position systems have been proposed to solve indoor positioning problems (e.g., Wi-Fi, Bluetooth, infrared, and RFID), but they come with challenges involving accuracy, installation, interference, and power consumption. We propose a next generation indoor localization recognition system called IP-PFM (Indoor Position based on Panorama Feature Matching) to overcome the limitations and problems of wireless technology-based approaches. By applying panorama image recognition technology, IP-PFM accurately pinpoints the indoor position. Further, IP-PFM also includes an indoor position system chat bot feature to automate chat in real-time with users - using the Line Bot application programming interface (API) - which allows a mobile user to post a picture taken with their smartphone. Upon receiving the picture, IP-PFM determines the location and plots it on the floor plan. We have implemented a prototype of IP-PFM to demonstrate the end-to-end functionality of the indoor localization recognition chat bot system ${ }^{1}$.
\end{abstract}

Keywords: Location-based service; indoor positioning; panoramic; image recognition; Line Bot.

\section{INTRODUCTION}

In recent years, positioning technology and location-based services (LBS) have become increasingly relevant to our lives, as they are used in navigation systems, such as Google Maps. Outdoor positioning technology is mostly ineffective for applications requiring indoor positioning systems (IPSs). However, IPSs may have high commercial value for use in department store merchandise shopping guidelines, home care service for seniors, airport station path guidance, and intelligent factory robot navigation are common applications

${ }^{1}$ DOI reference number: 10.18293/DMSVIVA2021-010
[1]. As a result, Google Maps is moving toward in the direction of implementing IPS, and recently provided real-time pictures of 13 Tokyo Metro stations to reduce the number of passengers who lost their way in complex underground tunnels. If indoor positioning system can be used in conjunction with the Internet of Things (IoT), it can provide a broader range and ubiquitous services. Many IPSs based on wireless technology (such as Wi-Fi and Bluetooth) are usually used only in particular situations. For example, the user may have to be a staff member of the site to access the area's Wi-Fi system for positioning, meaning that visitors could not use the same approach for positioning. IPSs contain many variant wireless parameters, such as Angle of Arrival (AoA), Time of Flight (ToF), Return Time of Flight (RTOF), and Receive Signal Strength (RSS), but these technologies have bottlenecks and blind spots [2]. Wireless IPSs also have problems with accuracy, installation, interference, and power consumption [3].

Because of the accuracy and time delay in IPSs, researchers initially used image data to build a reference model (including architectural models, floor plans, windows, doors, walkways, or other building parts) to make improvement happen [4]. Some of them used the characteristics of the current image to match the architectural reference model or the objects and features in the floor plan, and finally, they extrapolated the location of the camera [5].

A system with machine learning in the back-end combined with reliable image recognition IPS could solve the complex problem of blurred images due to focal length or light. Therefore, some scholars developed a framework based on two digital cameras to solve the problem of the blurred image [6]. When we enter an unfamiliar building, even if we have the building directory, we cannot quickly determine our location. Therefore, in this paper, we demonstrate how to use feature recognition technology to achieve indoor positioning and propose an indoor positioning method based on panoramic image recognition combined with a Line Bot to create an automatic indoor positioning robot system. Owing to the pop- 
ularity of mobile devices, we can take pictures anytime and anywhere, and users can shoot the image in front of them and upload it to a server instantly. After the images are processed by a server with panoramic photos in the database, we can obtain the user's location as well as the calculated distance and field of view to increase accuracy and user experience.

The structure of this paper is as follows. Section 2 lists some relevant researches that will be applied during the implementation stage. Section 3 introduces the detailed structure of the method proposed in this research and various calculation methods. Section 4 describes the experimental results. Section 5 shows the implementation and results of the relevant design methods. Section 6 summarizes and discusses future developments and applications of this paper.

\section{RELATED WORK}

This section elaborates on relevant technical applications and execution methods based on image feature matching. One approach is to apply Scale-Invariant Feature Transform (SIFT) [7] on a space vector to find the extreme point and extract its coordinates, image, and rotation invariant features through the algorithm to identify the object. The fault tolerance is quite high for light, noise, and error in the visual angle. In addition, the application of this algorithm is extensive. For these reasons, there are many improved feature recognition techniques that are designed based on SIFT. The feature recognition technology used in this article, Speeded Up Robust Features (SURF) [8], is also a SIFT-based scheme which is improved the performance with the use of the Hessian matrix to operate on the feature points. In terms of processing efficiency, SURF can achieve much higher speeds than SIFT, and it is more stable than SIFT in image transformation.

Salarian et al. [9] claimed that accurate information about the location and orientation of cameras in mobile devices is at the heart of the use of adaptive services, such as LBS. Most mobile devices rely on global positioning system (GPS) data, but the satellite's signal quality is not always good enough, which may lead to positioning errors. Therefore, they used SURF feature recognition technology on the image with the GPS tag image database to find the best match with the scene of the picture. However, this method compared the image with the general image data rather than the panoramic image; if there was no relevant information in the database, it was unable to make accurate corrections. As such, they constructed a location system by applying the technology of structure from motion (SfM) and a 3D camera position reconstruction model. In more than 170 sets of data experiments, the average error of their experimental results was less than five meters, which compares favorably with some other published methods. The disadvantage of this method is that its accuracy is related to the size of the database. If the information is incomplete, the error increases.

Xiao et al. [10] used the SUN360's panoramic image database and a Support Vector Machine (SVM) training model to enable the system to identify scenes and perspectives such as hospitals, restaurants, and theaters, as well as the orientations faced by observers. They trained two classifiers in the training model. The first was the scene classifier. For this classifier, the model trained m kinds of classifiers to train the experimental panoramic image database on m kinds of panoramic image categories. The second was a classifier of images with different angles of view. For this classifier, the same type of images with different angles of view were used for training. The number of different types of panoramic images was gradually increased to achieve effective classification results. The key limitation is that the author only classifies the panoramic image into four different types (symmetry, single axis symmetry, double axis symmetry, and full symmetry). Therefore, this approach to visual angle recognition has some problems. When encountering highly repeatable images in visual angle recognition and the angle of view is exactly on the axis of symmetry, it is easy to produce errors due to too high similarity, despite training through the SVM model.

Molnar and Kovacs [11] proposed a method for making panoramic images by using pixels that look for image boundaries and searching for overlapping areas to find the same boundaries for overlap. As their method does not need to grab feature points and feature comparisons, it can be run on lowresolution images. Because the red-green-blue (RGB) color in the pixel varies due to the brightness between different images, the RGB color was converted to grayscale before performing the calculations to prevent impacts on accuracy. They also use a graphics processing unit (GPU) acceleration to improve in the experiment significantly; however, the authors mentioned that if the range of the video arc is too large, there would be a reduction in accuracy.

Kawaji et al. [12] applied Locality Sensitive Hash (LSH) method to speed up the image matching process in their proposed indoor positioning system, and used the Railway Museum with 426 omnidirectional panoramas and 1067 supplementary images for the experimental study. LSH is a powerful approach to find the nearest neighbor technique, but it has a hash collision problem which is inevitable. Further, the proposed system does not consider images with different angles of view such that it needs many omnidirectional panoramas and supplementary images to construct a confidence database.

Huang et al. [13] proposed a Sorting Hat method to filter out unrelated element pairs to accommodate the mobile device environment where the image matching process needs to achieve a good design meeting the performance requirements. Sorting Hat starts the matching process from the innermost ring (containing the most critical features to determine similarity between two images) toward the outer ring. However, the proposed method was not practical and cost-effective because it requires extra sensors and computation power to calculate the user's current viewing direction.

\section{IP-PFM}

Figure 9 shows the process for the proposed IP-PFM which uses feature recognition technology, SURF, to calculate the characteristics of the user input image and find the features and the panoramic image in the database to perform a match calculation. Then, based on the matching results, the distance and where the user is facing will be estimated. Finally, the user's possible location will be computed and marked on the map. TABLE I shows the symbols related to IP-PFM algorithms and calculations. 


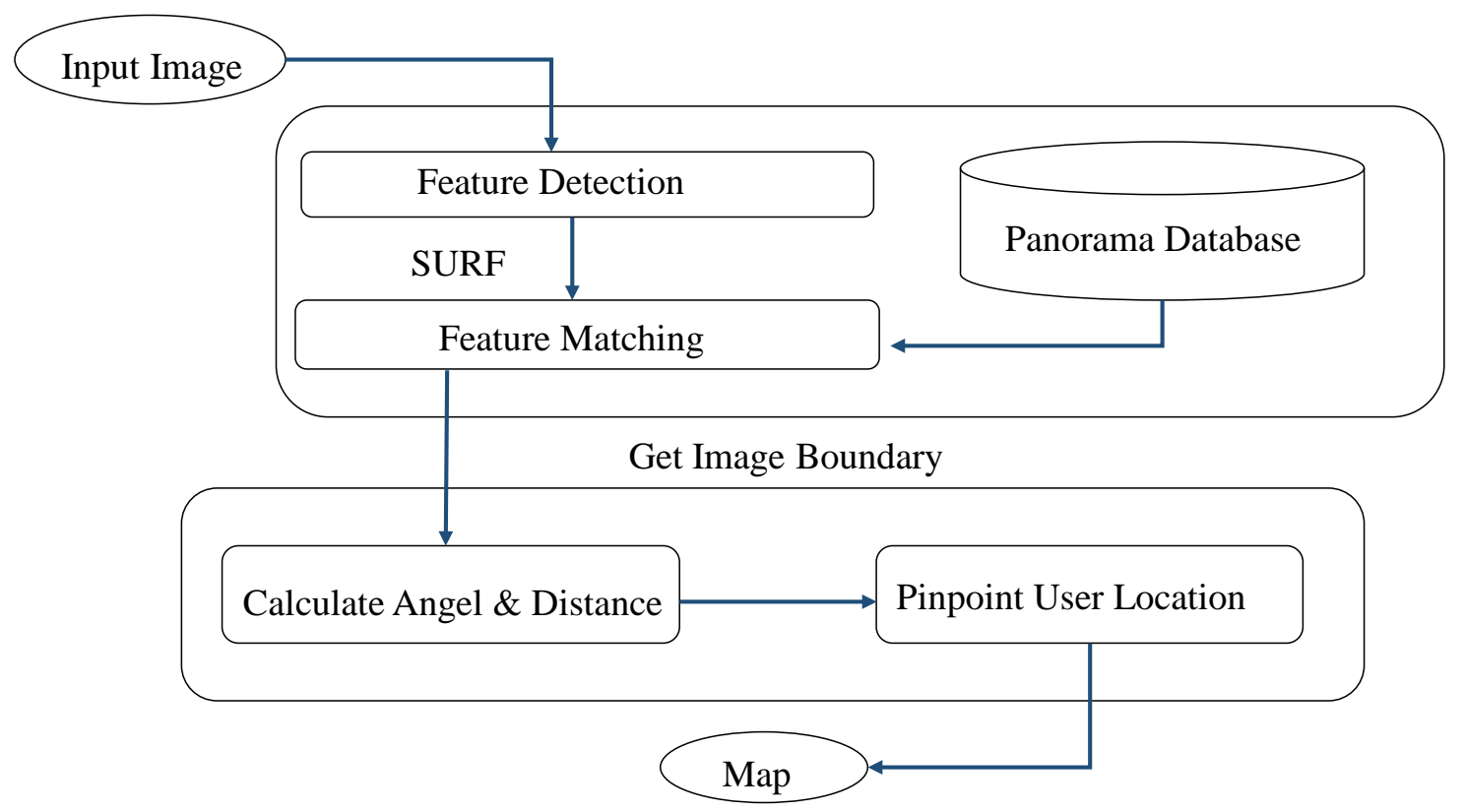

Figure 1. IP-IMP System architecture

TABLE I. Algorithmic symbols table

\begin{tabular}{cl}
\hline Symbol & \multicolumn{1}{c}{ Description } \\
\hline$N$ & Number of panoramic images in the da- \\
$\alpha$ & tabase \\
$\alpha_{\max }$ & Match point number \\
$M$ & Max match point number \\
$B_{r}$ & Right boundary \\
$B_{l}$ & Left boundary \\
$V_{r}$ & Matched range for four vertices \\
{$[i]$} & The panorama in the database \\
$p$ & The image the user inputs \\
$C p$ & The coordinate information of the \\
$C u$ & matched panorama on the map \\
& The coordinate information of the user on \\
$D$ & the map \\
$\theta l$ & Prediction distance \\
$\theta_{r}$ & Angle of left boundary \\
$\theta$ & Angle of right boundary \\
$\omega$ & Angle of viewpoint \\
$W_{c}$ & Width of panorama \\
$W_{60}$ & Width in the panorama for the picture \\
$\theta_{l}$ & taken at the panorama center \\
$\theta_{r}$ & Width in the panorama for the picture \\
$\theta$ & taken at 60 cm from the panorama center \\
\hline${ }_{n}$ we detail the IP-PFM implementation as following
\end{tabular}

Now, we detail the IP-PFM implementation as following.

\section{A. Pairs of Images and Panoramas}

We use SURF to detect feature points of the photos inputted by users, and IP-IMP compares these with panoramic photos in the database (as shown in Algorithm 1: line 2 8). Subsequently, the input photos that correspond to the panoramic photo are determined. IP-PFM uses the best matching panoramic image to compute the left boundary $\left(\mathrm{B}_{\mathrm{l}}\right)$ and right boundary $\left(B_{r}\right)$ of the range of the user input picture in the panoramic image (as shown in Algorithm 1: line 9 11). In other words, the position information of the image input by the user is calculated to find where the user is situated in the panoramic image. As shown in IP-IMP finds the left and right boundaries of the range (green frame) of the user input image in the panoramic image.

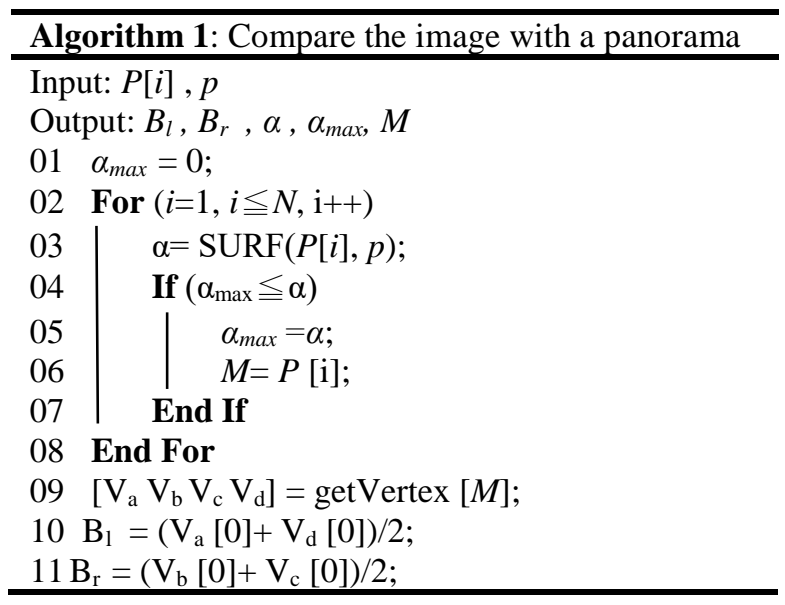

Algorithm 1 Match user pictures and panoramic functions

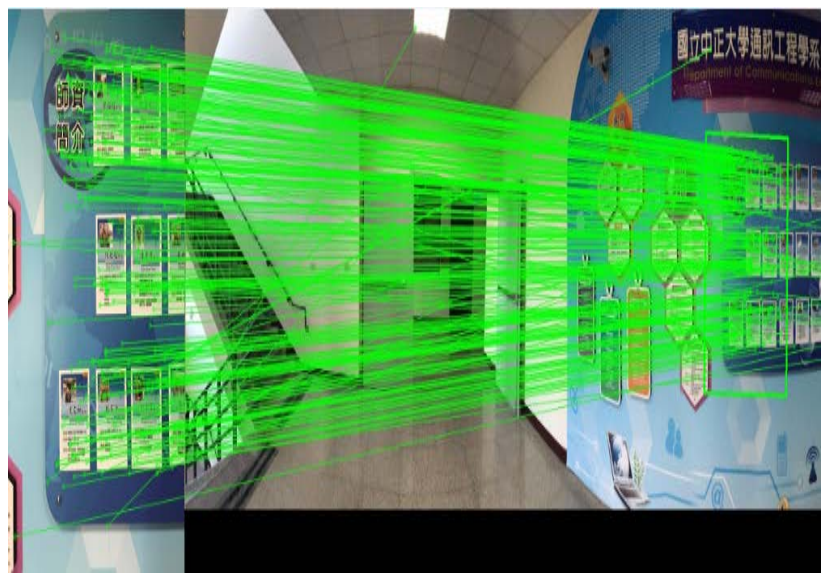

Figure 2 Photo by SURF and boundary calculation 


\section{B. Angle and distance calculations}

Next step is to calculate the user's position information (distance from the center of the panoramic image and facing angle). The center of the panoramic image is the location where the panoramic image was taken, and the distance from the center of the panoramic image is the predicted value after calculations are performed by the system. IP-PFM determines how far the user must be from this position to take this picture. The facing angle means that the leftmost side of the panoramic image is set to 0 degrees. This means that IP-PFM needs to know which angle in the panoramic image where the user sees this scene.

Using the left and right boundaries obtained in Algorithm 1 , the range of the user-shot image in the panoramic image is calculated using the left and right boundaries. Then, the distance between the user's shooting position and the matching panoramic image center and the orientation (expressed in degrees) are calculated. The angle can be obtained in Eq. (1) and illustrated the result of the inference in Figure 3.

$$
\theta=\left\lfloor\left(B_{l} / \omega\right) \times 360+\left(B_{r} / \omega\right) \times 360\right\rfloor / 2
$$

$\mathrm{B}_{\mathrm{l}}$ and $\mathrm{B}_{\mathrm{r}}$ are the left and right borders of the panoramic image, respectively, which are results calculated using Algorithm 1 . When the leftmost side of the panoramic image is 0 degrees, the user's facing angle is obtained through Eq. (1). Example: If width of panorama image $(\boldsymbol{\omega})=4,096, \mathrm{~B}_{\mathrm{l}}=$ 2,000, and $B_{r}=2,096$, as found in Algorithm 1, then the user's facing angle is calculated to be $180^{\circ}$.

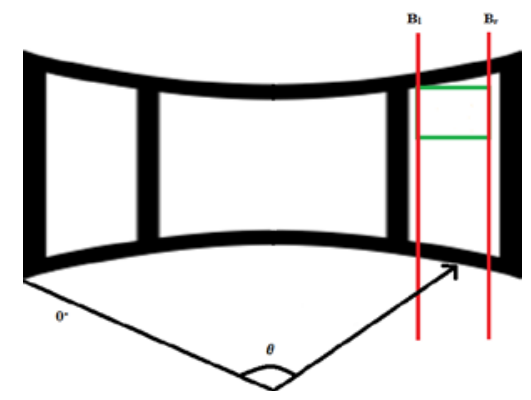

Figure 3 Angle calculation schematic

The distance is obtained by Eq. (2) , which is derived from Figure 4.

$$
\mathrm{D}=60 \times\left[\frac{\mathrm{W}_{C}}{\left(W_{60}-W_{C}\right)}\right] \times \frac{\left|B_{l}-B_{r}\right|-W_{C}}{W_{C}}
$$

$W_{c}$ is the width of the picture taken by the user in the panoramic image; $W_{60}$ is the width of the range of the photograph taken at $60 \mathrm{~cm}$ from the panoramic image center, and 60 is the calculated reference length.

With the shot remaining the same and without zooming, the field of view for taking the photo at the same location will be static. That is, $\mathrm{W}_{\mathrm{c}}$ and $\mathrm{W}_{60}$ are constant values. In the camera lens used in this paper, these two values are approximately 1,600 and 1,200, respectively. As we have the $\mathrm{B}_{\mathrm{l}}$ and $\mathrm{B}_{\mathrm{r}}$ calculated by Algorithm 1, we can calculate that the distance through Eq. (2). In other words, the two above equations can thus obtain the face of the user's photograph and the distance between the location of the user's photo and the center of the matching panoramic image.

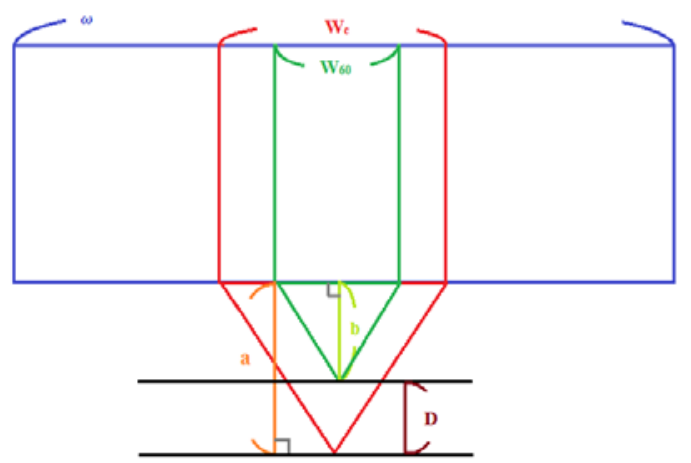

Figure 4 Diagram for the distance calculation

\section{Output map and indicate user location}

After Eq. (1) calculates the user's facing angle $\theta$ and Eq. (2) calculates the distance D between the location of the user's captured image and the center of the panoramic image, the user's location can be estimated based on these two pieces of information and marked on the map. As shown in Figure 5, $C_{p}$ is the coordinate on the map with the center position of the matched panoramic image, and $\mathrm{D}$ is the estimated distance between the user and the center position of the panoramic image calculated by Equation (2). Here, $\theta$ is the estimated angle. Combining these two results and cooperating with the scale of the map, the coordinate position $\mathrm{C}_{\mathrm{u}}$ of the user's image can be obtained through Eq. (3). Finally, we mark the location of $\mathrm{C}_{\mathrm{u}}$ on the map.

$$
\mathrm{C}_{u}=C_{p}+\left[\cos \theta^{*} D, \sin \theta * D\right]
$$

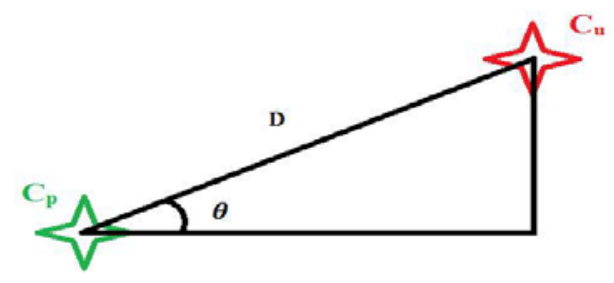

Figure 5 Panorama image center and user coordinate relationship

\section{EXPERIMENT}

This experiment uses the feature recognition technology, SURF, which is a mature technology. It is used not only for image comparison but also in facial recognition technologyrelated research. As such, this paper adopts the current open source code of SURF in OpenCV 2.4.11. We choose a SURF identifier to provide a stable feature recognition function and then use the algorithm in IP-PFM to obtain the required information.

The client app developed and tested through Line Bot achieved the purpose of this paper. The image is sent to the server from the user, and the result is returned to the user through the calculation of the server. The indoor building 
directory is displayed in the app. The map also pinpoints the user's location. The experiment environment is shown in TABLE II.

TABLE II. EXPERIMENTAL ENVIRONMENT

\begin{tabular}{ll}
\hline \multicolumn{1}{c}{ SW/HW } & \multicolumn{1}{c}{ Description } \\
\hline Processor & Intel Core i7-3770 \\
RAM & 16GB \\
Operating System & MS Windows 7 (64-bit) \\
$\begin{array}{l}\text { Programming } \\
\text { Language }\end{array}$ & Java \\
Camera & 12 megapixels; Wide-angle: \\
\hline
\end{tabular}

\section{A. Distance from shooting location to panoramic center} and error relationship

In a single scene, we shoot multiple images at different distances from the center of the panorama and use these images as system inputs. Then, we calculate the distance between the location where the image was shot and the center of the panorama. Figure 6 shows the results and the actual errors. Among them, -120, -60, 0, 60, and 120 are the actual distances from the center of the panorama when shooting the image. Negative values represent moving forward from the center (i.e., the field of view of the image is narrower and the coverage is smaller), and positive values represent moving backwards from the center (i.e., the field of view of the image becomes wider and covers a wider range). This also applies in terms of the distance estimation error.

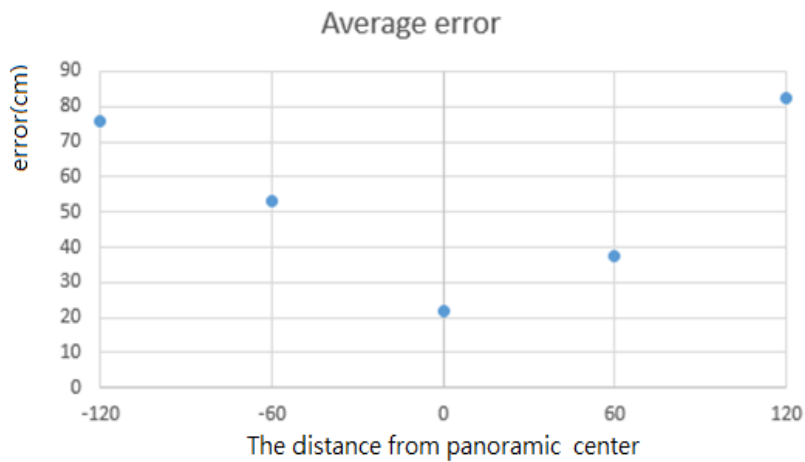

Figure 6 The shooting position and the panoramic center distance pair error graph

After the system calculations, an image taken farther from the center of the matching panoramic image will have a larger error. Conversely, if the scene image is taken closer to the center of the panoramic image, there will be a smaller error value after the calculation. Overall, the average error value was within one meter.

\section{B. System prediction position and actual position error value}

In this experimental simulation, 61 sets of panoramic images were set as benchmarks, and 70 scene images were samples. Regarding the estimation errors in this experiment after system calculations, Figure 7 shows the results obtained by using 10 images captured in the center of the panorama in different scenes. The green points represent the center of the panorama corresponding to the test image, which is where the image was taken. The red points mark the system's final estimation of the location where this image was taken. TABLE III shows the error values of these 10 different calculation results. The minimum value of the error value was $4 \mathrm{~cm}$ and the maximum value was $123 \mathrm{~cm}$. This scale of error is considered acceptable for an indoor setting.

\section{The relationship between the number of panoramic images and the error in the database}

From Figure 8, we know that the more panoramic images there are in the database, the smaller the distance calculation error. This means that the more complete the panoramic image data in the database, the higher the accuracy of the system. This also solves the problem of increased error because the shooting position is further away from the panoramic center. As long as the number of panoramic images increases, the distance between the location of the captured image and the center of the corresponding panoramic image can be reduced, thereby decreasing the error value of the system judgment.

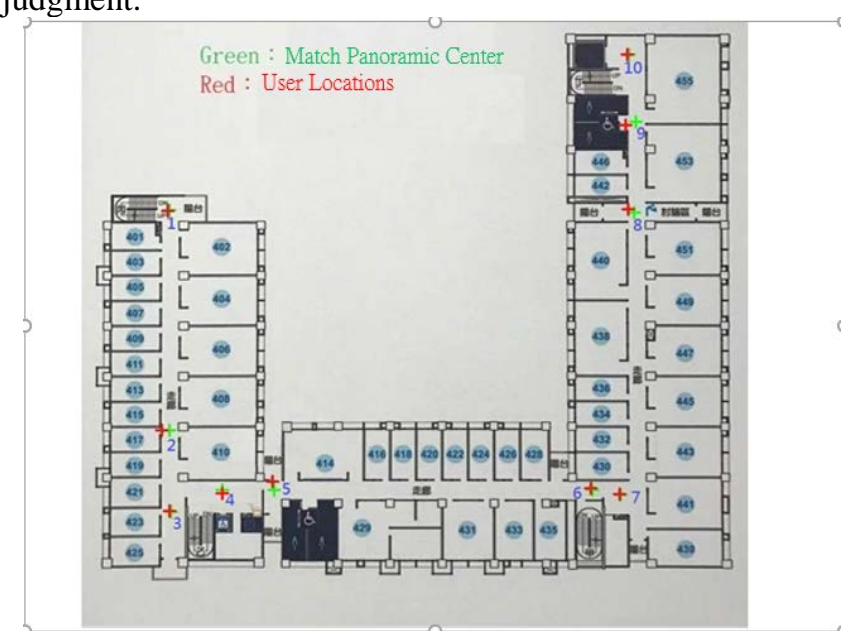

Figure 7 User location and panoramic map

TABLE III. ERROR VALUE TABLE

\begin{tabular}{cccc}
\hline Number & $\begin{array}{c}\text { Error } \\
(\mathbf{c m})\end{array}$ & Number & $\begin{array}{c}\text { Error } \\
(\mathbf{c m})\end{array}$ \\
\hline 1 & 21 & 6 & 21 \\
2 & 79 & 7 & 4 \\
3 & 14 & 8 & 68 \\
4 & 47 & 9 & 123 \\
5 & 96 & 10 & 17 \\
\hline
\end{tabular}

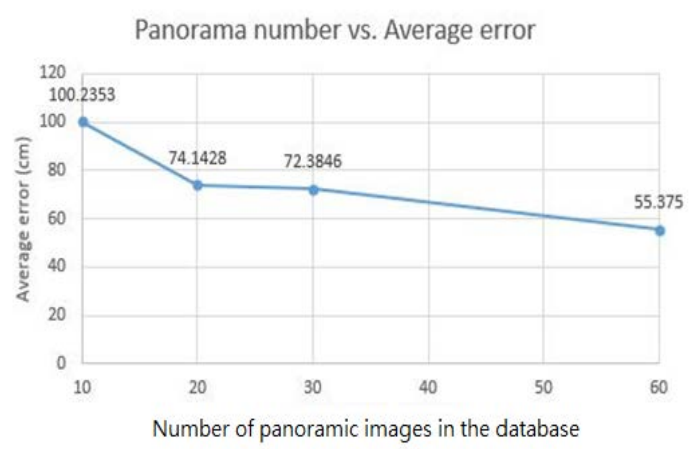

Figure 8 Panorama quantity vs. average errors 


\section{SYSTEM IMPLEMENTATION}

The paper combines Line Bot with the method of image calculation and location estimation to make a usable indoor positioning robot system. Figure 9 shows the system with Line Bot as a bridge between the user and the IP-PFM. The first step is that the user sends a photo to Line Bot. Then the message is obtained by the server with Webhook, and the system can download the user transmitted information (photos) and use the method proposed in this paper to calculate the data. Finally, the system sends the map link to Line Bot, and the user receives the result.

Figure 10 is the conversation between the user and Line Bot implemented in the IP-PFM system. After the user sends a photo to Line Bot, the system receives the photo through Webhook and saves it. The bot sends a message to inform the user that the image has been successfully received and calculation is started. Upon finishing the calculation, the bot uploads the URL that contains the user's location picture. Finally, it sends the link URL message to the user. The user could use the URL to obtain the building directory. Figure 11 shows the map that users can see after connecting to the link.

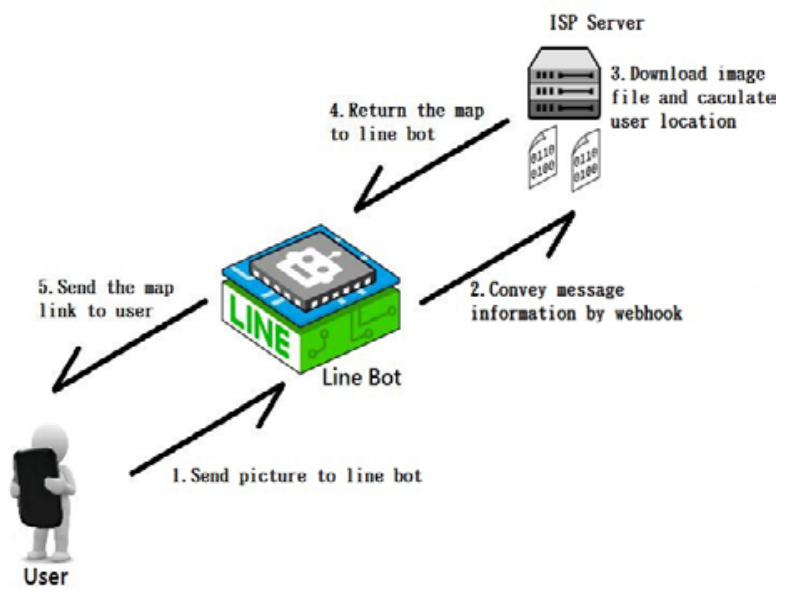

Figure 9. IP-PFM system architecture.

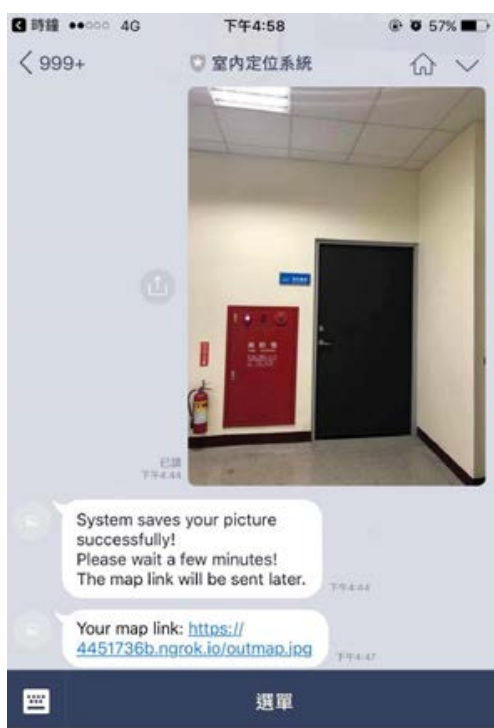

Figure 10 User and Line Bot conversation.

\section{CONCLUSION}

This paper proposes a method, IP-PFM, for indoor positioning based on panoramic images and image recognition, which it combines with Line Bot. The complete procedure requires a user to input a photo of a scene, which is compared to the characteristics of panoramic images in the database. The system then estimates where the user has taken

the photo and where the user is located by calculating the distance and direction between the user's input and the matching panoramic image. Finally, the system transmits the result back to the user by Line Bot.

This practical system can be customized according to local conditions. In the future, it may be possible to apply this method to robots to collect panoramic maps of buildings and 2D plane maps and to store these data directly in the computer system storage, which would be very useful for indoor positioning. In terms of computing speed, the experiment in this paper used only a central processing unit (CPU), and the processing was slow for image recognition. Adding a GPU may increase the processing speed so that users can access information faster.

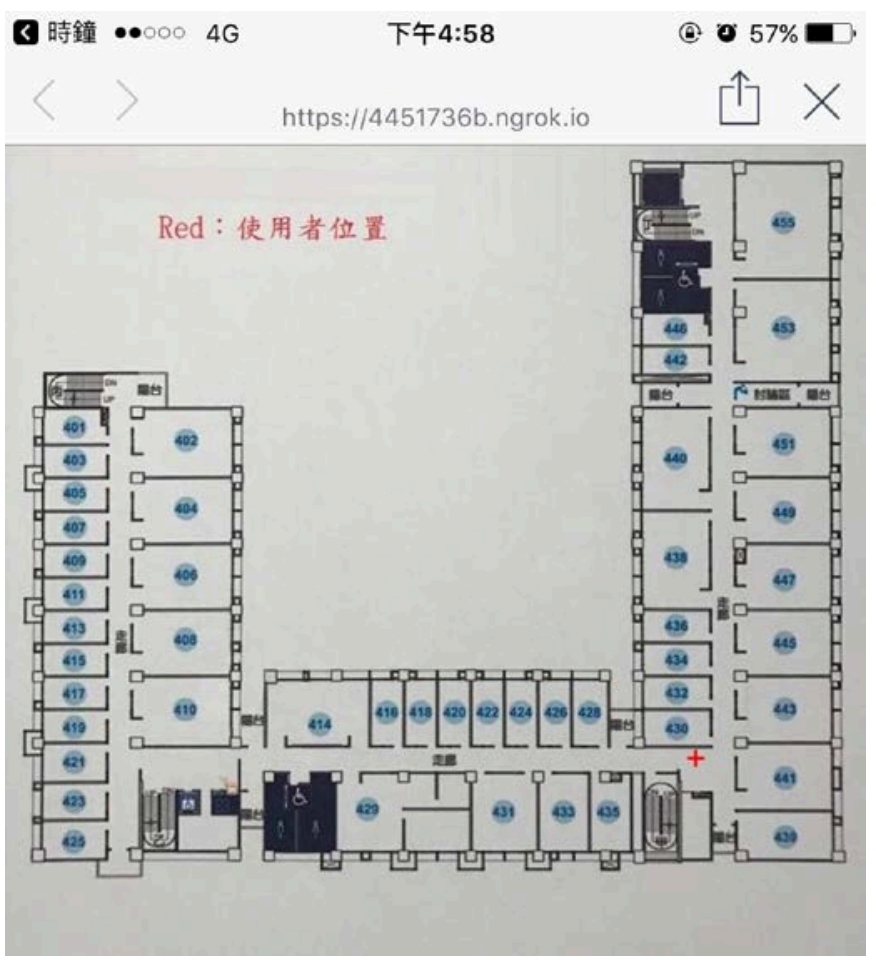

Figure 11 URL link image sent by the system.

\section{ACKNOWLEDGEMENTS}

This research was partially supported by Ministry of Science and Technology (MOST), Taiwan, R.O.C., under the grant number MOST 109-2221-E-005 -047 and 108-2221-E-194 010 -MY2.

\section{REFERENCES}

[1] A. Yassin, Y. Nasser, M. Awad, A. Al-Dubai, R. Liu, C. Yuen, R. Raulefs, and E. Aboutanios, "Recent Advances in Indoor Localization: A Survey on Theoretical Approaches and Applications,” IEEE Communications Surveys \& Tutorials, vol. 19, no. 2, pp. 1327-1346, 2017.

[2] F. Zafari, A. Gkelias, and K. K. Leung, "A Survey of Indoor Localization Systems and Technologies," IEEE Communications Surveys \& Tutorials, vol. 21, no. 3, pp. 2568-2599, 2019. 
[3] F. Dwiyasa and M.-H. Lim, "A survey of problems and approaches in wireless-based indoor positioning,” 2016 International Conference on Indoor Positioning and Indoor Navigation (IPIN), Oct. 2016.

[4] H. An and N. Moon, "Image-based positioning system using LED Beacon based on IoT central management," Multimedia Tools and Applications, Nov. 2020.

[5] M. Shahjalal, M. T. Hossan, M. K. Hasan, M. Z. Chowdhury, N. T. Le and Y. M. Jang, “An Implementation Approach and Performance Analysis of Image Sensor Based Multilateral Indoor Localization and Navigation System," Wireless Communications and Mobile Computing, vol. 2018, pp. 1-13, Oct. 2018.

6] P. Pascacio, S. Casteleyn, J. Torres-Sospedra, E. S. Lohan, and J. Nurmi, "Collaborative Indoor Positioning Systems: A Systematic Review,” Sensors, vol. 21, no. 3, p. 1002, Feb. 2021.

[7] D. G. Lowe, "Object recognition from local scale-invariant features," Proceedings of the Seventh IEEE International Conference on Computer Vision, 1999.

[8] H. Bay, A. Ess, T. Tuytelaars, and L. Van Gool, "Speeded-Up Robust Features (SURF)," Computer Vision and Image Understanding, vol. 110, no. 3, pp. 346-359, Jun. 2008.

[9] M. Salarian, N. Ileiv, and R. Ansari, “Accurate Image Based Localization by Applying SFM and Coordinate System Registration,” 2016 IEEE International Symposium on Multimedia (ISM), Dec. 2016.

[10] Jianxiong Xiao, K. A. Ehinger, A. Oliva, and A. Torralba, "Recognizing scene viewpoint using panoramic place representation,” 2012 IEEE Conference on Computer Vision and Pattern Recognition, Jun. 2012.

[11] F. Molnar and A. Kovacs, "360-Degree image stitching with GPU support,” 2016 IEEE 20th Jubilee International Conference on Intelligent Engineering Systems (INES), Jun. 2016.

[12] H. Kawaji, K. Hatada, T. Yamasaki, and K. Aizawa, "Image-based indoor positioning system: fast image matching using omnidirectional panoramic images," Proceedings of the 1st ACM international workshop on Multimodal pervasive video analysis - MPVA '10, 2010.

[13] J.-Y. Huang, S.-H. Lee, and C.-H. Tsai, "A fast image matching technique for the panoramic-based localization,” 2016 IEEE/ACIS 15th International Conference on Computer and Information Science (ICIS), Jun. 2016. 